\title{
Hyaluronic Acid as a Marker of Sinusoidal Obstruction Syndrome after Oxaliplatin-based Chemotherapy for Colorectal Liver Metastases: Don't Forget the Tumor
}

\author{
Nuh N. Rahbari, MD ${ }^{1}$ and Juergen Weitz, MD, MSc ${ }^{2}$ \\ ${ }^{1}$ Edwin L. Steele Laboratory for Tumor Biology, Department of Radiation Oncology, Massachusetts General Hospital and \\ Harvard Medical School, Boston, MA; ${ }^{2}$ Department of Visceral, Thoracic, and Vascular Surgery, University Hospital Carl \\ Gustav Carus Technical University Dresden, Dresden, Germany
}

A few decades ago, patients with colorectal cancer liver metastases were considered incurable, and treatment was mainly supportive. Treatment options for patients with metastatic colorectal cancer have changed greatly since then, as has the outcome of these patients. Although in the past survival of these patients was within the range of a few months, with no 5-year survivors, current studies report median survival rates of 20-25 months and 5-year survival rates of $30-60 \% .^{1-4}$ What has happened?

Numerous studies have taught us that in selected patients, surgical resection of metastases prolongs survival. As hepatic resection has evolved to a safe procedure even in patients requiring extended, complex, and/or two-stage resection for complete tumor clearance, its use has increased and has added to the improved long-term outcome. However, only approximately $15 \%$ to $20 \%$ of patients are initially amenable to potentially curative resection; therefore, further factors must have played a role in the marked improvement in survival. The development and increased use of effective chemotherapeutic regimens has contributed significantly to the treatment results we are currently documenting for metastatic colorectal cancer. Several phase III trials proved that the addition of oxaliplatin or irinotecan to the backbone of 5-fluorouracil (5-FU) and folinic acid is able to achieve objective response rates of $40-50 \% .^{5-8}$ The antitumor activity of these combined chemotherapy regimens has clearly improved median survival rates compared to historic data

(C) Society of Surgical Oncology 2013

Published Online: 6 March 2013

J. Weitz, MD, MSc

e-mail: juergen.weitz@uniklinikum-dresden.de of patients who received 5-FU monotherapy, which was the standard of care in the late 1980s. Moreover, these improved regimens rendered the disease of a higher proportion of patients resectable. Resectability rates in firstline chemotherapy trials with palliative intent range from 5 to $15 \%$ and go up to $30 \%$ in patients with more limited yet still unresectable disease receiving chemotherapy for the intent of downstaging. ${ }^{9}$ Furthermore, the addition of biological agents such as bevacizumab, a monoclonal antibody against the vascular endothelial growth factor (VEGF), and cetuximab or panitumumab, monoclonal antibodies against the epidermal growth factor receptor, seem to further enhance response and resectability rates. ${ }^{10}$

Although the effectiveness of modern chemotherapy protocols in patients with metastatic colorectal cancer is undisputed, issues associated with these regimens, such as acquired resistance and increased toxicity, remain important future challenges for interdisciplinary management. In particular, liver toxicity related to the administration of irinotecan- and oxaliplatin-containing regimens represents a significant concern; their development may interfere with the planned treatment concept. It has been demonstrated that preoperative chemotherapy correlates with significantly higher perioperative complications. ${ }^{11,12}$ Furthermore, the development of histological alterations in the liver parenchyma may impede the antitumor effects of systemic therapies. ${ }^{13}$ The pathological changes of the liver are specific to the administered agents. Irinotecan has been associated with chemotherapy-associated steatohepatitis. Oxaliplatin has been more frequently reported to cause hepatic injury, which typically manifests as sinusoidal obstruction syndrome (SOS) and occurs in up to $50 \%$ of patients who receive an oxaliplatin-based regimen. SOS is considered to 
result from severe toxic injury to the sinusoidal endothelial cells. It is characterized histologically by sinusoidal dilatation and congestion, perisinusoidal hemorrhage and fibrosis, nodular regeneration, and loss of hepatocytes. ${ }^{14}$ Fibrosis can also affect the centrilobar vein (veno-occlusive disease) and contributes to the congestion of the liver, which causes its typical blueish appearance and may result in portal hypertension. Despite the clinical relevance of SOS, to date, there is no validated biomarker to assess the development, presence, or severity of SOS. Such a biomarker is needed for (early) noninvasive diagnosis of SOS, which could prompt clinicians to adjust the chemotherapy regimen and the intended operative procedure.

In the present issue of Annals of Surgical Oncology, van den Broek et al. investigated hyaluronic acid (HA) as a marker of SOS in 40 colorectal cancer patients undergoing resection of liver metastases who received systemic chemotherapy protocols including oxaliplatin. ${ }^{15}$ The authors report higher systemic HA levels in patients with moderate and severe SOS compared to those who developed mild and no SOS. Using equations and HA levels measured in the hepatic vein, hepatic artery, and portal vein, they calculated the fractional extraction of HA by the liver and the splanchnic area. The calculations of the hepatic and splanchnic fractional extraction of HA did not yield a significant difference, though the authors report nonsignificant trends toward a net hepatic uptake of HA in patients with mild or no SOS and a net release of HA in patients with moderate or severe SOS.

Why HA as a marker of SOS? As mentioned above, the initial step in the pathogenesis of SOS is currently believed to take place at the level of the sinusoidal endothelial cells. For this reason, it is reasonable and logical to evaluate parameters of endothelial function as early markers of SOS. The glycosaminoglycan HA is an important component of the extracellular matrix in various tissues. Its degradation occurs almost exclusively in the sinusoidal endothelial cells, which take up HA by receptor-mediated endocytosis. Because of its high turnover, clearance of HA is therefore considered to be a direct indicator of sinusoidal endothelial function. The available data on HA levels after ischemia-reperfusion injury and veno-occlusive disease in patients with bone marrow transplantation further support the notion of HA as a promising biomarker for the diagnosis and severity of SOS in patients with liver metastases receiving oxaliplatin-based chemotherapy. ${ }^{16,17}$ However, these studies did not include patients with a significant burden of solid tumors.

It is a puzzling yet interesting finding that systemic HA levels are increased in patients with moderate to severe SOS despite similar fractional extraction of HA in the liver and splanchnic area. In their discussion, the authors address several possible explanations for their results, such as the small sample size and the resulting lack of power; the placement of portosystemic shunts due to development of portal hypertension in patients with moderate or severe SOS; and enhanced production of HA at extrahepatic sites. However, one should note that HA is a major extracellular matrix component of various solid malignancies, including colorectal cancer, and its expression has been linked to disease progression. ${ }^{18-20}$ The release of HA from the tumor therefore contributes to HA levels measured in the circulation. With respect to the present study, it is also important to note that $78 \%$ of patients had synchronous metastases, and a liver-first approach was chosen in $22 \%$-that is, the primary tumor as a source of HA was still in situ and contributed to plasma HA concentrations. In case of patients with (low) rectal cancer, HA release by the primary tumor could not have been controlled for by sampling from the portal vein. To rule out the influence of HA expression and release by the primary and metastatic tumors on systemic HA levels and HA fractional extractions tumor-related factors such as tumor burden and location, HA expression in the tumor, presence of the primary tumor and the extent of response to therapy need to be considered. Even more importantly, blood sampling sites must be standardized: samples ought to be consistently collected from the hepatic vein of the tumor-bearing liver or the non-tumor-bearing liver.

It is still unclear how anti-VEGF therapy affects the development and severity of SOS. There is mounting evidence that the addition of bevacizumab to oxaliplatin-based chemotherapy may decrease the incidence of higher-grade sinusoidal injury. ${ }^{21-23}$ A potential impact of bevacizumab treatment on oxaliplatin-induced SOS is backed by studies that demonstrate an involvement of angiogenesis pathways in this disease. ${ }^{24,25}$ Although the present study does not demonstrate a protective influence of bevacizumab on SOS development, it is of importance that $88 \%$ of patients received oxaliplatin-based chemotherapy together with bevacizumab. It is thus difficult to evaluate how anti-VEGF therapy affected the HA levels measured in the present study. In order to make generalizable statements on the value of HA as a marker of SOS in patients with colorectal liver metastases, further data are needed on patients who received oxaliplatin-based chemotherapy without bevacizumab.

The results of this study are important because they support the hypothesis that systemic indicators of sinusoidal endothelial function may serve as markers of SOS in colorectal cancer patients receiving oxaliplatin-based chemotherapy. The study design includes measurements of HA in systemic circulation as well as its fractional extraction, which may allow conclusions to be drawn on the source and clearance of HA. However, in order to prove the specificity of measured HA levels for oxaliplatininduced injury of liver sinusoidal endothelial cells, further 
studies are required that take into account the tumor as a source of systemic HA. The management of patients with marginal resectable hepatic metastases of colorectal cancer remains complex and needs to be tailored to the individual patient by an experienced multidisciplinary team.

\section{REFERENCES}

1. Wilson SM, Adson MA. Surgical treatment of hepatic metastases from colorectal cancers. Arch Surg. 1976;111:330-4.

2. Bengtsson G, Carlsson G, Hafstrom L, et al. Natural history of patients with untreated liver metastases from colorectal cancer. Am J Surg. 1981;141:586-9.

3. Schmoll HJ, Cunningham D, Sobrero A, et al. Cediranib with mFOLFOX6 versus bevacizumab with mFOLFOX6 as first-line treatment for patients with advanced colorectal cancer: a doubleblind, randomized phase III study (HORIZON III). J Clin Oncol. 2012;30:3588-95.

4. Reissfelder C, Rahbari NN, Koch M, et al. Validation of prognostic scoring systems for patients undergoing resection of colorectal cancer liver metastases. Ann Surg Oncol. 2009;16: 3279-88.

5. de Gramont A, Figer A, Seymour M, et al. Leucovorin and fluorouracil with or without oxaliplatin as first-line treatment in advanced colorectal cancer. J Clin Oncol. 2000;18:2938-47.

6. Giacchetti S, Perpoint B, Zidani R, et al. Phase III multicenter randomized trial of oxaliplatin added to chronomodulated fluorouracil-leucovorin as first-line treatment of metastatic colorectal cancer. J Clin Oncol. 2000;18:136-47.

7. Douillard JY, Cunningham D, Roth AD, et al. Irinotecan combined with fluorouracil compared with fluorouracil alone as firstline treatment for metastatic colorectal cancer: a multicentre randomised trial. Lancet. 2000;355(9209):1041-7.

8. Saltz LB, Cox JV, Blanke C, et al. Irinotecan plus fluorouracil and leucovorin for metastatic colorectal cancer. Irinotecan Study Group. N Engl J Med. 2000;343:905-14.

9. Folprecht G, Grothey A, Alberts S, et al. Neoadjuvant treatment of unresectable colorectal liver metastases: correlation between tumour response and resection rates. Ann Oncol. 2005;16:1311-9.

10. Folprecht G, Gruenberger T, Bechstein WO, et al. Tumour response and secondary resectability of colorectal liver metastases following neoadjuvant chemotherapy with cetuximab: the CELIM randomised phase 2 trial. Lancet Oncol. 2010;11:38-47.

11. Nordlinger B, Sorbye H, Glimelius B, et al. Perioperative chemotherapy with FOLFOX 4 and surgery versus surgery alone for resectable liver metastases from colorectal cancer (EORTC Intergroup trial 40983): a randomised controlled trial. Lancet. 2008;371(9617):1007-16.
12. Karoui M, Penna C, Amin-Hashem M, et al. Influence of preoperative chemotherapy on the risk of major hepatectomy for colorectal liver metastases. Ann Surg. 2006;243:1-7.

13. Vreuls CP, van den Broek MA, Winstanley A, et al. Hepatic sinusoidal obstruction syndrome (SOS) reduces the effect of oxaliplatin in colorectal liver metastases. Histopathology. 2012;61:314-8.

14. Rubbia-Brandt L. Sinusoidal obstruction syndrome. Clin Liver Dis. 2010;14:651-68.

15. van den Broek M, Vreuls C, Winstanley A, et al. Hyaluronic acid as a marker of hepatic sinusoidal obstruction syndrome secondary to oxaliplatin-based chemotherapy in patients with colorectal liver metastases. Ann Surg Oncol. 2013. doi:10.1245/s10434013-2915-8.

16. Fried MW, Duncan A, Soroka S, et al. Serum hyaluronic acid in patients with veno-occlusive disease following bone marrow transplantation. Bone Marrow Transpl. 2001;27:635-9.

17. Itasaka H, Suehiro T, Wakiyama S, et al. Significance of hyaluronic acid for evaluation of hepatic endothelial cell damage after cold preservation/reperfusion. J Surg Res. 1995;59:589-95.

18. Lugli A, Zlobec I, Gunthert U, et al. Overexpression of the receptor for hyaluronic acid mediated motility is an independent adverse prognostic factor in colorectal cancer. Mod Pathol. 2006;19:1302-9.

19. Llaneza A, Vizoso F, Rodriguez JC, et al. Hyaluronic acid as prognostic marker in resectable colorectal cancer. $\mathrm{Br} J$ Surg. 2000;87:1690-6.

20. Ropponen K, Tammi M, Parkkinen J, et al. Tumor cell-associated hyaluronan as an unfavorable prognostic factor in colorectal cancer. Cancer Res. 1998;58:342-7.

21. Kishi Y, Zorzi D, Contreras CM, et al. Extended preoperative chemotherapy does not improve pathologic response and increases postoperative liver insufficiency after hepatic resection for colorectal liver metastases. Ann Surg Oncol. 2010;17:2870-6.

22. Rubbia-Brandt L, Lauwers GY, Wang H, et al. Sinusoidal obstruction syndrome and nodular regenerative hyperplasia are frequent oxaliplatin-associated liver lesions and partially prevented by bevacizumab in patients with hepatic colorectal metastasis. Histopathology. 2010;56:430-9.

23. Tamandl D, Klinger M, Eipeldauer S, et al. Sinusoidal obstruction syndrome impairs long-term outcome of colorectal liver metastases treated with resection after neoadjuvant chemotherapy. Ann Surg Oncol. 2011;18:421-30.

24. Agostini J, Benoist S, Seman M, et al. Identification of molecular pathways involved in oxaliplatin-associated sinusoidal dilatation. J Hepatol. 2012;56:869-76.

25. Rubbia-Brandt L, Tauzin S, Brezault C, et al. Gene expression profiling provides insights into pathways of oxaliplatin-related sinusoidal obstruction syndrome in humans. Mol Cancer Ther. 2011;10:687-96. 